\title{
Green function for Klein-Gordon-Dirac equation
}

\author{
Vasyl KOVALCHUK \\ Institute of Fundamental Technological Research, Polish Academy of Sciences, \\ Świętokrzyska 21., 00-049 Warsaw, Poland \\ E-mail:vkoval@ippt.gov.pl
}

This article is part of the Proceedings titled "Geometrical Mathods in Physics: Bialowieza XXI and XXII"

\begin{abstract}
The Green function for Klein-Gordon-Dirac equation is obtained. The case with the dominating Klein-Gordon term is considered. There seems to be a formal analogy between our problem and a certain problem for a 4-dimensional particle moving in the external field. The explicit relations between the wave function, Green function and initial conditions are established with the help of the $T$-exponent formalism.
\end{abstract}

\section{Introduction}

The idea of this paper is actually a by-product of the paper [1], where we discussed some physical justification and a general solution for the Klein-Gordon-Dirac (KGD) equation. This is a linear differential equation with constant coefficients which is obtained by superposing Dirac and d'Alembert operators and which has originally arisen from the $U(2,2)$ ruled gauge model of spinorial geometrodynamics in a natural and logical way $[2,3]$. The case considered here is in a sense complementary to the one studied in [1]-[3]. Namely, restrictions on the correlations between coefficients of the KGD equation, which are necessary for the existence of real non-negative solutions for the particle mass (non-tachyonic situation) are just opposite to those in [1]-[3]. In other words, we are describing the case with the dominating Klein-Gordon term; this situation is opposite to the case studied in [1]-[3], where the Dirac term was dominating.

\section{Green function for KGD equation}

Let us consider the Klein-Gordon-Dirac equation [1]-[3]

$$
i v \gamma^{\mu} \partial_{\mu} \Psi-\omega \Psi=u g^{\mu \nu} \partial_{\mu} \partial_{\nu} \Psi, \quad \mu=\overline{0,3}
$$

derivable from the corresponding KGD Lagrangian

$$
£=u g^{\mu \nu} \partial_{\mu} \bar{\Psi} \partial_{\nu} \Psi+\frac{i v}{2}\left(\bar{\Psi} \gamma^{\mu} \partial_{\mu} \Psi-\partial_{\mu} \bar{\Psi} \gamma^{\mu} \Psi\right)-\omega \bar{\Psi} \Psi,
$$


where $u, v$, and $\omega$ are some real constants, $\gamma^{\mu}$ are Dirac matrices satisfying the Clifford rules $\left\{\gamma^{\mu}, \gamma^{\nu}\right\}=2 g^{\mu \nu}, g^{\mu \nu}$ is the metric tensor, which in the specially relativistic (SR) limit equals $\eta^{\mu \nu}$, i.e, the flat metric tensor on space-time manifold $M$ with the signature $(+,-,-,-)$ and constant coefficients; $\bar{\Psi}=\Psi^{+} \gamma^{0}$ is the Dirac conjugated wave function. For completeness of description we should add the following initial conditions:

$$
\left.\Psi\left(x^{\mu}\right)\right|_{t=t_{0}}=\Psi_{0}\left(\vec{r}, t_{0}\right),\left.\quad \frac{\partial \Psi\left(x^{\mu}\right)}{\partial t}\right|_{t=t_{0}}=\Phi_{0}\left(\vec{r}, t_{0}\right) .
$$

From the equation (2.1) we can define the KGD operator $\widehat{K}_{G D}$ as follows:

$$
\widehat{K}_{G D}=u g^{\mu \nu} \partial_{\mu} \partial_{\nu}-i v \gamma^{\mu} \partial_{\mu}+\omega \equiv u g^{\mu \nu}\left(\partial_{\mu}-i a_{\mu}\right)\left(\partial_{\nu}-i a_{\nu}\right)+\widetilde{\omega},
$$

where $a_{\mu}=(v / 2 u) g_{\mu \nu} \gamma^{\nu}$ and $\widetilde{\omega}=\omega+u g^{\mu \nu} a_{\mu} a_{\nu}-i u g^{\mu \nu}\left(\partial_{\mu} a_{\nu}\right)$. In the specially relativistic limit we have $\widetilde{\omega}=\omega+v^{2} / 4 u$. We can define the momenta $\hat{p}_{\mu}=-i \partial_{\mu}$ (we use the natural system of units, i.e., $e=c=\hbar=1$ ); then

$$
\widehat{K}_{G D}=-u g^{\mu \nu}\left(\hat{p}_{\mu}-a_{\mu}\right)\left(\hat{p}_{\nu}-a_{\nu}\right)+\widetilde{\omega} \equiv-u \widehat{K}_{G},
$$

where $\widehat{K}_{G}=g^{\mu \nu}\left(\hat{p}_{\mu}-a_{\mu}\right)\left(\hat{p}_{\nu}-a_{\nu}\right)+m^{2}$ is some Klein-Gordon operator for the 3-dimensional particle of the mass $m^{2}=-\widetilde{\omega} / u$ (in the SR case $m^{2}=-\left(4 u \omega+v^{2}\right) / 4 u^{2}$ ) in the external field $a_{\mu}$. For the existence of real non-negative solutions for $m^{2}$ (non-tachyonic situation) we should have in the SR limit $(u \omega<0) \wedge\left(v^{2} \leq 4|u \omega|\right)$. As we have already said in the Introduction, this situation is complementary to the conditions which we had in [1]-[3], i.e., $(u \omega \geq 0, \forall v) \vee\left(u \omega<0, v^{2} \geq 4|u \omega|\right)$. Now we can define the Green function for the KGD equation as follows:

$$
\widehat{K}_{G D} D\left(x, x_{0}\right)=\delta^{(4)}\left(x-x_{0}\right) \quad \text { or } \quad\left(\widehat{H}_{x}+\frac{m}{2}\right) D\left(x, x_{0}\right)=-\frac{1}{2 m u} \delta^{(4)}\left(x-x_{0}\right),
$$

where $\widehat{H}_{x}=(1 / 2 m) g^{\mu \nu}\left(\hat{p}_{\mu}-a_{\mu}\right)\left(\hat{p}_{\nu}-a_{\nu}\right)$ is the Hamiltonian operator for the 4-dimensional particle in the external field $a_{\mu}$. At this stage we consider a general form of the metric tensor $g^{\mu \nu}(x)$, i.e., non-constant one; then the external field $a_{\mu}(x)$ is also non-constant and can be formally interpreted as some "electromagnetic" field. This analogy is, of course, only superficial and is broken in the SR case, when $g^{\mu \nu}(x)$ becomes $\eta^{\mu \nu}$ and $a_{\mu}(x)$ becomes a constant field $a_{\mu}$. Later on we will consider only the SR situation.

The Green function can be obtained formally from (2.6) as follows:

$$
D\left(x, x_{0}\right)=-\frac{1}{2 m u}\left(\widehat{H}_{x}+\frac{m}{2}\right)^{-1} \delta^{(4)}\left(x-x_{0}\right) .
$$

We can use the Feynman representation of the inverse operator (as, e.g., in [4]), i.e.,

$$
\widehat{B}^{-1}= \pm \frac{1}{i} \int_{0}^{\infty} d s e^{-\epsilon s} e^{ \pm i s \widehat{B}}, \quad \epsilon \rightarrow 0
$$

to rewrite $(2.7)$ in the following form:

$$
D\left(x, x_{0}\right)=-\frac{i}{2 m u} \int_{0}^{\infty} d s e^{-i s \frac{m}{2}} Q\left(x, s ; x_{0}, 0\right),
$$


where $s$ is an evolution parameter (the proper time), $Q\left(x, s ; x_{0}, 0\right)=e^{-i s \widehat{H}_{x}} \delta^{(4)}\left(x-x_{0}\right)$ is the Green function for the corresponding stationary Schrödinger equation in 4-dimensional space:

$$
i \frac{\partial \varphi(x ; s)}{\partial s}=\widehat{H}_{x} \varphi(x ; s), \quad \widehat{H}_{x}=\frac{1}{2 m} \eta^{\mu \nu}\left(\hat{p}_{\mu}-a_{\mu}\right)\left(\hat{p}_{\nu}-a_{\nu}\right) .
$$

It is obtained by performing the differentiation over $s$ of $Q\left(x, s ; x_{0}, 0\right)$ :

$$
\left(i \frac{\partial}{\partial s}-\widehat{H}_{x}\right) Q\left(x, s ; x_{0}, 0\right)=i \delta^{(4)}\left(x-x_{0}\right) \delta(s) .
$$

For obtaining the explicit expression for the Green function $Q\left(x, s ; x_{0}, 0\right)$ we can use the following equality for operators $[4,5]$ :

$$
e^{\frac{\alpha}{2} \widehat{A}^{2}}=\frac{1}{\sqrt{2 \pi}} \int_{-\infty}^{+\infty} d \xi \exp \left(-\frac{1}{2} \xi^{2} \pm \xi \sqrt{\alpha} \widehat{A}\right)
$$

then:

$$
Q\left(x, s ; x_{0}, 0\right)=-\frac{i m^{2}}{4 \pi^{2} s^{2}} \exp \left\{\frac{i m}{2 s}\left[\left(t-t_{0}\right)^{2}-\left(\vec{r}-\vec{r}_{0}\right)^{2}\right]+i a_{\mu}\left(x^{\mu}-x_{0}^{\mu}\right)\right\} .
$$

Finally, after substituting (2.13) in (2.9) and introducing a new variable $\xi=m / s$, we represent the total Green function $D\left(x, x_{0}\right)$ in the following form:

$$
D\left(x, x_{0}\right)=-\frac{G(y, z)}{8 \pi^{2} u} \exp \left\{i a_{\mu}\left(x^{\mu}-x_{0}^{\mu}\right)\right\}, G(y, z)=\int_{0}^{\infty} d \xi \exp \left\{-\frac{1}{2}\left(y \xi+\frac{z}{\xi}\right)\right\}
$$

where $y=i\left[\left(\vec{r}-\vec{r}_{0}\right)^{2}-\left(t-t_{0}\right)^{2}\right], z=i m^{2}$. For performing the integration procedure in (2.14) we can construct formally the differential equation for which our function $G(y, z)$ is a solution. It turns out that this is the modified Bessel equation $\partial^{2} G / \partial z^{2}-$ $(y / 4 z) G=0$, which has the following solution (the condition for mixed second derivatives, i.e., $\partial^{2} G(y, z) / \partial y \partial z=\partial^{2} G(y, z) / \partial z \partial y=(1 / 4) G(y, z)$, is already taken into account): $G(y, z)=a \delta(-i y)+b \sqrt{z / y} Z_{1}(\sqrt{y z})$, where $a, b$ are constants, $Z_{1}(z)$ is either the modified Bessel function of the first kind $I_{1}(z)$ or the second kind $K_{1}(z)[6]$.

We can notice that for a massless "particle" we have $G(y, 0)=\int_{0}^{\infty} d \xi \exp \{-y \xi / 2\}=$ $2 \pi \delta(-i y)+2 \mathcal{P} / y$ (Sokhotskyi formulae), where $\mathcal{P} / y$ is a generalized function (just like $\delta$ function) and the symbol $\mathcal{P}$ itself stands for the integration in the principal value meaning [5]. For the modified Bessel functions we can write the approximate formulae $I_{1}(z) \approx z / 2$ and $K_{1}(z) \approx 1 / z$ for $|z| \ll 1$. Hence, we can choose the modified Bessel function of the second kind $K_{1}$ and our constants $a$ and $b$ are as follows: $a=2 \pi, b=2$. Then we have $\sqrt{y z}=$ $m \sqrt{\left(t-t_{0}\right)^{2}-\left(\vec{r}-\vec{r}_{0}\right)^{2}}$ for $\left(t-t_{0}\right)^{2}>\left(\vec{r}-\vec{r}_{0}\right)^{2}$ and $\sqrt{y z}=i m \sqrt{\left(\vec{r}-\vec{r}_{0}\right)^{2}-\left(t-t_{0}\right)^{2}}$ for $\left(t-t_{0}\right)^{2}<\left(\vec{r}-\vec{r}_{0}\right)^{2}$. So for the latter case it is convenient not to use the modified Bessel functions $K_{1}$ but the Hankel functions $H_{1}^{(1)}$ or $H_{1}^{(2)}$, the Bessel functions of the third kind, which are interrelated as follows: $(2 / \pi) K_{1}(i x)=-H_{1}^{(1)}(-x)=-H_{1}^{(2)}(x)$. 
Finally, the Green function $D\left(x, x_{0}\right)$ has the following form:

$$
\begin{aligned}
D\left(x, x_{0}\right)= & -\frac{\exp \left\{i a_{\mu}\left(x^{\mu}-x_{0}^{\mu}\right)\right\}}{4 \pi u}\left[\delta\left(\left(t-t_{0}\right)^{2}-\left(\vec{r}-\vec{r}_{0}\right)^{2}\right)+\right. \\
& +\left\{\begin{array}{c}
\frac{m}{\pi} \frac{K_{1}\left(m \sqrt{\left(t-t_{0}\right)^{2}-\left(\vec{r}-\vec{r}_{0}\right)^{2}}\right)}{\sqrt{\left(t-t_{0}\right)^{2}-\left(\vec{r}-\vec{r}_{0}\right)^{2}}}, \text { if }\left(t-t_{0}\right)^{2}>\left(\vec{r}-\vec{r}_{0}\right)^{2} \\
-\frac{i m}{2} \frac{H_{1}^{(2)}\left(m \sqrt{\left(\vec{r}-\vec{r}_{0}\right)^{2}-\left(t-t_{0}\right)^{2}}\right)}{\sqrt{\left(\vec{r}-\vec{r}_{0}\right)^{2}-\left(t-t_{0}\right)^{2}}}, \text { if }\left(t-t_{0}\right)^{2}<\left(\vec{r}-\vec{r}_{0}\right)^{2}
\end{array}\right] .
\end{aligned}
$$

\section{General solution of the KGD equation}

Now let us consider the two-component wave function $\vec{\Psi}=\left(\Psi_{1}, \Psi_{2}\right)^{T}, \Psi_{1}=\Psi, \Psi_{2}=$ $i \partial \Psi / \partial t$. Then our second-order differential equation (2.1) (in the SR case) becomes the system of two first-order ones:

$$
\begin{aligned}
& i \frac{\partial \Psi_{1}}{\partial t}=\Psi_{2}, \\
& i \frac{\partial \Psi_{2}}{\partial t}=\left(-\triangle+i \frac{v}{u} \gamma^{j} \nabla_{j}+\frac{\omega}{u}\right) \Psi_{1}-\frac{v}{u} \gamma^{0} \Psi_{2} .
\end{aligned}
$$

In the symbolic way we may rewrite the previous equations as follows:

$$
i \frac{\partial \vec{\Psi}}{\partial t}=\widehat{L}_{\vec{r}}(t) \vec{\Psi}, \quad \widehat{L}_{\vec{r}}(t)=\left[\begin{array}{cc}
0 & 1 \\
-\triangle+\frac{i v}{u} \gamma^{j} \nabla_{j}+\frac{\omega}{u} & -\frac{v}{u} \gamma^{0}
\end{array}\right] .
$$

The initial conditions (2.3) for this symbolic Schrödinger equation can be rewritten as follows: $\vec{\Psi}_{0}\left(\vec{r}, t_{0}\right)=\left(\Psi_{0}\left(\vec{r}, t_{0}\right), \Phi_{0}\left(\vec{r}, t_{0}\right)\right)^{T}$. Then we can use the $T$-exponent method for describing the Green function of Schrödinger equation [4]. First of all, the differential equation (3.2) can be rewritten as the integro-differential one:

$$
\vec{\Psi}(\vec{r}, t)=\vec{\Psi}_{0}\left(\vec{r}, t_{0}\right)+\frac{1}{i} \int_{t_{0}}^{t} d t^{\prime} \widehat{L}_{\vec{r}}\left(t^{\prime}\right) \vec{\Psi}\left(\vec{r}, t^{\prime}\right)
$$

Using the iteration method we can solve this equation and introduce the Green function in the matrix form as follows:

$$
\begin{aligned}
& \vec{\Psi}(\vec{r}, t)=\int d^{3} \vec{r}_{0} \widehat{G}\left(\vec{r}, t ; \vec{r}_{0}, t_{0}\right) \vec{\Psi}_{0}\left(\vec{r}_{0}, t_{0}\right), \\
& \widehat{G}\left(\vec{r}, t ; \vec{r}_{0}, t_{0}\right)=\theta\left(t-t_{0}\right) \delta\left(\vec{r}-\vec{r}_{0}\right) T \exp \left\{-i \int_{t_{0}}^{t} d t^{\prime} \widehat{L}_{\vec{r}}\left(t^{\prime}\right)\right\} .
\end{aligned}
$$

The $T$-exponent operator here is understood as a series:

$$
T \exp \left\{-i \int_{t_{0}}^{t} d t^{\prime} \widehat{L}_{\vec{r}}\left(t^{\prime}\right)\right\}=1+\sum_{n \geq 1} \int_{t_{0}}^{t} d t_{1} \int_{t_{0}}^{t_{1}} d t_{2} \ldots \int_{t_{0}}^{t_{n-1}} d t_{n} \widehat{L}_{\vec{r}}\left(t_{1}\right) \widehat{L}_{\vec{r}}\left(t_{2}\right) \ldots \widehat{L}_{\vec{r}}\left(t_{n}\right)
$$

and the symbol $T$ itself stands for the chronological multiplication of operators, e.g., for two operators $\widehat{A}(t)$ and $\widehat{B}\left(t^{\prime}\right)$ we have the following rule:

$$
T\left(\widehat{A}(t) \widehat{B}\left(t^{\prime}\right)\right)=\theta\left(t-t^{\prime}\right) \widehat{A}(t) \widehat{B}\left(t^{\prime}\right)+\theta\left(t^{\prime}-t\right) \widehat{B}\left(t^{\prime}\right) \widehat{A}(t) .
$$


The equation (3.4a) can be rewritten in the explicit form:

$$
\begin{aligned}
& \Psi(\vec{r}, t)=\int d^{3} \vec{r}_{0}\left[G_{11}\left(\vec{r}, t ; \vec{r}_{0}, t_{0}\right) \Psi_{0}\left(\vec{r}_{0}, t_{0}\right)+i G_{12}\left(\vec{r}, t ; \vec{r}_{0}, t_{0}\right) \Phi_{0}\left(\vec{r}_{0}, t_{0}\right)\right], \\
& i \frac{\partial \Psi(\vec{r}, t)}{\partial t}=\int d^{3} \vec{r}_{0}\left[G_{21}\left(\vec{r}, t ; \vec{r}_{0}, t_{0}\right) \Psi_{0}\left(\vec{r}_{0}, t_{0}\right)+i G_{22}\left(\vec{r}, t ; \vec{r}_{0}, t_{0}\right) \Phi_{0}\left(\vec{r}_{0}, t_{0}\right)\right],
\end{aligned}
$$

i.e., for defining $\Psi(\vec{r}, t)$ at any time instant $t$ it is necessary to know only the Green function matrix components $G_{11}\left(\vec{r}, t ; \vec{r}_{0}, t_{0}\right)$ and $G_{12}\left(\vec{r}, t ; \vec{r}_{0}, t_{0}\right)$. With the help of differentiation over $t$ in $(3.4 \mathrm{~b})$ we can find the following equation for the Green function $\widehat{G}$ :

$$
\frac{\partial \widehat{G}}{\partial t}=-i \widehat{L}_{\vec{r}}(t) \widehat{G}+\delta\left(t-t_{0}\right) \delta\left(\vec{r}-\vec{r}_{0}\right) \widehat{I}
$$

where $\widehat{I}$ is the identity matrix. In the explicit form it is a system of four equations:

$$
\begin{aligned}
i \frac{\partial G_{11}}{\partial t} & =G_{21}+i \delta\left(t-t_{0}\right) \delta\left(\vec{r}-\vec{r}_{0}\right), \\
i \frac{\partial G_{12}}{\partial t} & =G_{22}, \\
i \frac{\partial G_{21}}{\partial t} & =\left[-\Delta+\frac{i v}{u} \gamma^{j} \nabla_{j}+\frac{\omega}{u}\right] G_{11}-\frac{v}{u} \gamma^{0} G_{21}, \\
i \frac{\partial G_{22}}{\partial t} & =\left[-\triangle+\frac{i v}{u} \gamma^{j} \nabla_{j}+\frac{\omega}{u}\right] G_{12}-\frac{v}{u} \gamma^{0} G_{22}+i \delta\left(t-t_{0}\right) \delta\left(\vec{r}-\vec{r}_{0}\right) .
\end{aligned}
$$

From the equations (3.8a) and (3.8c) we can see that the matrix component $G_{11}$ satisfies the following equation:

$$
\widehat{K}_{G D} G_{11}=u \delta\left(\vec{r}-\vec{r}_{0}\right) \frac{\partial}{\partial t} \delta\left(t-t_{0}\right)-i v \gamma^{0} \delta\left(\vec{r}-\vec{r}_{0}\right) \delta\left(t-t_{0}\right) .
$$

Equivalently, composing $(3.8 \mathrm{~b})$ and $(3.8 \mathrm{~d})$ we obtain

$$
\widehat{K}_{G D} G_{12}=-i u \delta\left(\vec{r}-\vec{r}_{0}\right) \delta\left(t-t_{0}\right) .
$$

The initial conditions for these equations, which can be obtained from (3.6), are as follows:

$$
G_{11}\left(\vec{r}, t_{0} ; \vec{r}_{0}, t_{0}\right)=\delta\left(\vec{r}-\vec{r}_{0}\right), \quad G_{12}\left(\vec{r}, t_{0} ; \vec{r}_{0}, t_{0}\right)=0 .
$$

The $G_{11}$ and $G_{12}$ are not independent functions. If we use the properties of the $\delta$-function in (3.9), then we may replace the differentiation over $t$ by the differentiation over $t_{0}$. We can notice that the KGD operator $\widehat{K}_{G D}$ and the operator $i \partial / \partial t_{0}+(v / u) \gamma^{0}$ are commuting because they act on different variables. Then we can write that

$$
G_{11}\left(\vec{r}, t ; \vec{r}_{0}, t_{0}\right)=\left[i \frac{\partial}{\partial t_{0}}+\frac{v}{u} \gamma^{0}\right] G_{12}\left(\vec{r}, t ; \vec{r}_{0}, t_{0}\right)
$$

Moreover, the equation (3.10) for the Green function $G_{12}\left(\vec{r}, t ; \vec{r}_{0}, t_{0}\right)$ is almost identical with the equation (2.6) for the KGD equation Green function $D\left(\vec{r}, t ; \vec{r}_{0}, t_{0}\right)$. Hence, if we define the retarded Green function for the KGD equation

$$
D_{\text {ret }}\left(\vec{r}, t ; \vec{r}_{0}, t_{0}\right)=\theta\left(t-t_{0}\right) D\left(\vec{r}, t ; \vec{r}_{0}, t_{0}\right),
$$


then we can write that $G_{12}\left(\vec{r}, t ; \vec{r}_{0}, t_{0}\right)=-i u D_{\text {ret }}\left(\vec{r}, t ; \vec{r}_{0}, t_{0}\right)$.

The general solution of the KGD equation (2.1) (for the time instant $t>t_{0}$ ) with the initial conditions (2.3) is as follows:

$$
\begin{aligned}
\Psi(\vec{r}, t) & =\int d^{3} \vec{r}_{0}\left(u \frac{\partial}{\partial t_{0}}-i v \gamma^{0}\right) D_{\text {ret }}\left(\vec{r}, t ; \vec{r}_{0}, t_{0}\right) \Psi_{0}\left(\vec{r}_{0}, t_{0}\right) \\
& +u \int d^{3} \vec{r}_{0} D_{\text {ret }}\left(\vec{r}, t ; \vec{r}_{0}, t_{0}\right) \Phi_{0}\left(\vec{r}_{0}, t_{0}\right) .
\end{aligned}
$$

\section{Conclusions}

We have found the general solution for the KGD equation with the help of the Green function method. The KGD operator has been reduced to some extent to the usual KleinGordon operator, i.e., we supposed that the Klein-Gordon term in (2.1) was dominating. This was possible due to special relations between the coefficients of KGD equation. These relations are complementary to the ones in [1], where we have found the general solution for KGD equation as a superposition of two Dirac plane harmonic waves with different masses. In $[2,3]$ it has been shown that the appearance of two mass shells in the general solution not only is not undesirable but even can help explain, for example, a mysterious kinship between heavy leptons and their neutrinos or the corresponding pairing between quarks. Otherwise, the mass splitting $\triangle m=m_{+}-m_{-}$could be very large (then perhaps it is too difficult to excite the $m_{+}$-states) or very small (then perhaps it is below the present accuracy of our experiments) for not being found in the experimental way.

Acknowledgments. I would like to thank Professor Jan J. Sławianowski for supporting the idea of this article and valuable help in its realization. I also want to thank the Organizers of the XXI Workshop on Geometrical Methods in Physics (30 June - 6 July, 2002, Białowieża, Poland) for inviting me to the meeting, This work has been partially supported by the Committee of Scientific Research's (KBN) grant 8T07A04720.

\section{References}

[1] Sławianowski J J and Kovalchuk V, Klein-Gordon-Dirac Equation: Physical Justification and Quantization Attempts, Rep. on Math. Phys. 49 (2002), 249-257.

[2] Sławianowski J J, U(2,2)-Invariant Spinorial Geometrodynamics, Rep. on Math. Phys. 38 (1996), 375-397.

[3] Sławianowski J J, Internal Symmetries of Geometrodynamical Models, Rep. on Math. Phys. 48 (2000), 103-114.

[4] Blazhyevskyi L F, Operator Methods in Quantum Theory, Lviv University Press, Lviv, 1993 (in Ukrainian).

[5] Svidzynskyi A, Mathematical Methods of Theoretical Physics, Olena Teliga Publishing House, Kyiv, 1998 (in Ukrainian).

[6] Janke E, Emde F and Lösch F, Special Functions, Nauka, Moscow, 1977 (in Russian). 